\title{
PARAMETRIC FIELD MODELING FOR THE LHC MAIN MAGNETS IN OPERATING CONDITIONS
}

\author{
M. Di Castro ${ }^{1,2}$, D. Sernelius ${ }^{1,3}$, L. Bottura ${ }^{1}$, L. Deniau ${ }^{1}$, N. Sammut $^{1}$, S. Sanfilippo ${ }^{1}$, \\ W. Venturini Delsolaro ${ }^{1}$ \\ ${ }^{1}$ CERN, AT Department, 1211 Geneva 23, Switzerland \\ ${ }^{2}$ Universita` di Napoli “Federico II", Via Claudio 21, 80125, Napoli, Italy \\ ${ }^{3}$ Linköpings Universitet, Department of Physics and Measurement Technology, Linköping, Sweden
}

\begin{abstract}
The first beam injections and current ramps in the LHC will require a prediction of the settings of the magnet current as well as the main correctors. For this reason we are developing a parametric model of the magnetic field generated by the LHC magnets that will provide the field dependence on current, ramp-rate, time, and history. The model of the field is fitted on magnetic field measurements performed during the acceptance tests of the magnets before their installation in the machine. In this paper we summarize the different steps necessary to select the relevant data and identify the parameters: the data extraction, the filtering and the validation of the measurements, and the fitting procedure that is used to obtain the parameters from the experimental results. The main result reported is a summary of the value of the parameters obtained with the above procedure, and describing the behavior of the magnetic field in the LHC main dipoles and quadrupoles.
\end{abstract}

\section{INTRODUCTION}

The Large Hadron Collider (LHC) now under installation at CERN will provide proton-proton collisions with a centre-of-mass energy of $14 \mathrm{TeV}$. In order to achieve this design energy within the constraint of the $27 \mathrm{~km}$ LEP tunnel, the main bending magnets must provide a dipole field of $8.33 \mathrm{~T}$ while the main focusing magnets have to generate a quadrupole magnetic field at operational conditions of $223 \mathrm{~T} / \mathrm{m}$. The first beam injections and current ramps in the LHC will require a prediction of the settings of the main ring powering circuits as well as the main correctors. Taking into account the tight requirement of the field errors and the fact that only $20 \%$ of the magnets were magnetically measured at operational conditions, a parametric magnetic field model for all the population of the magnets is necessary. The algorithm that generates settings based on all the information available on the magnetic field generated by the ring magnet is the Field Description for the LHC (FiDeL). The main aim of FiDeL is to provide the integrated transfer function (TF) along the magnet's length (integral field vs. current) in a form suitable for inversion (current vs. integral field) for each powering circuit in the LHC by using a parametric model of the magnetic field. In addition, for the ring main magnets and main quadrupoles and dipoles, the parametric field model will provide a prediction of the field errors during the ramp to be used to set the corrector circuits that compensate for these field errors. The field model is based on magnetic field measurements performed on magnets at warm and operational conditions during the acceptance tests before their installation in the machine. In this paper a summary of the different steps necessary to select the relevant data from the raw cold measurements is given as well as the different steps necessary to identify the parameters for the running of the machine. The fitting procedures, based on developed mathematical models, used to extract the parameters from the experimental results are presented. A summary of the values of the parameters obtained is also given.

\section{DATA EXTRACTION: FILTERING AND VALIDATION}

All the magnets for the LHC were magnetically measured in industry at room temperature to monitor their production and the quality [1]. At CERN measurements have been performed in cryogenic conditions on a fraction of the magnet population [2]. The number of measurement entries during these series tests is estimated to have reached 3,500,000 raw entries. For the main dipoles and quadrupoles a measurement system based on twin rotating coils has been used to investigate the static and some of the dynamic effects [3]. Within the vast amount of measurements there exists erroneous data stemming from different error sources. A large task has been to investigate and identify this erroneous data and then clean the data by correcting or removing the records in question. Different tools and methods, depending on magnet and measurement type, have been used to identify the errors in the data. For the main dipoles and quadrupoles a semi-automatic system has been developed to analyze the data from the twin rotating coil measurements. The system fetches the raw measurement data from the database, calculates the field and field errors, summarizes the information and displays it in the form of an HTML-page containing key values and plots. The HTML format allows swift visual detection of abnormalities and errors in the data, and is also platform independent. The system gets all the necessary magnet analysis information from a settings file designated to each magnet. The file contains pertinent information such as what measurement runs are to be chosen and if any current-specific, time-specific, or coil-specific measurement points should be disregarded. The design of the informatic system makes it possible to generate the 
whole set of clean data by having access to all the settings files of the magnets, the analysis system and the database with raw measurements. The data is validated by comparing to expected values derived from design specifications and measurements, while keeping the measurement uncertainties in mind. When the measurement data have been validated, they are filtered into a reference table format common to all the different magnet types. The content of this reference table is then applied to the field model and is used to determine the magnet specific model parameters.

\section{FITTING MODELS}

The models for the static and dynamic magnetic field description of the LHC were defined and studied in the past [4]. The static magnetic field components represent the foundation of the model since they provide an experimentally reproducible mathematical description of the current dependent behavior of the superconducting magnets.

For the static transfer function of both the main field and the multipoles $\left(c_{n}\right)$, a 10-parameter model was built:

$$
\begin{aligned}
& T F=\gamma_{m} \pm \mu_{m}\left(\frac{I_{i n j}}{I}\right)^{2-p_{m}}\left(\frac{I_{c}-I}{I_{c}-I_{i n j}}\right)^{q_{m}}\left(\frac{T_{\infty}^{1.7}-T^{1.7}}{T_{c o}^{1.7}-T_{\text {meas }}^{1.7}}\right)^{h_{m}}+\sum_{i=1}^{N} \sigma_{m}^{i} \Sigma\left(I, S_{m}, I_{0 m}^{i}, I_{n o m}\right)+\rho_{m}\left(\frac{I_{i n j}}{I}\right)^{r_{m}} \\
& c_{n}^{d . c .}=\gamma_{n} \pm \mu_{n}\left(\frac{I_{i n j}}{I}\right)^{2-p_{n}}\left(\frac{I_{c}-I}{I_{c}-I_{i n j}}\right)^{q_{n}}\left(\frac{T_{c o}^{1.7}-T^{1.7}}{T_{c o}^{1.7}-T_{\text {meas }}^{1.7}}\right)^{h_{n}}+\sum_{i=1}^{N} \sigma_{n}^{j} \Sigma\left(I, S_{n}^{i}, I_{0 n}^{i}, I_{n o m}\right)+\rho_{n}\left(\frac{I_{i m j}}{I}\right)^{r_{n}}
\end{aligned}
$$

where $\mathrm{N}$ is typically 1 or 2 depending of the complexity of the geometry of the iron yoke and

$\sum\left(I, S, I_{0}, I_{n o m}\right)=\frac{1}{\pi} \tan ^{-1}\left(S\left(\frac{I-I_{0}}{I_{\text {nom }}}\right)\right)+\frac{1}{2}$.

$I_{i n j}$ and $I_{n o m}$ are the currents at injection and nominal conditions; $I_{c}(15000 \mathrm{~A})$ and $T_{c o}(9.5 \mathrm{~K})$ are constants, $T_{\text {meas }}$ is the measurement's temperature equal to $1.9 \mathrm{~K}$. This model takes into account physical contributions such as the geometric component that is due to the deviation between the conductor position in the real coil winding and the ideal distribution of current; the $D C$ magnetization due to the persistent currents in the superconducting filaments; the saturation of the iron yoke surrounding the coils; the displacement of the cables in the coil cross-section; and the residual magnetization of magnetic parts in the cold mass. Concerning field errors, another correction needs to be implemented due to the beam screen contribution [4]. Since the cold measurements were performed without the beam screen, the values of the geometric component obtained from the warm-cold correlation must be corrected. Table 1 provides a summary of all the parameters used for the static model as well as their meanings.

Fig. 1 shows an example of the static fit model applied on the average of all the transfer functions (ramp up) for the main quadrupoles measured at cold conditions.
Table 1: Summary of the fitting parameters of the static field model components ( 1 unit is equal to $10^{-4}$ of the main field).

\begin{tabular}{|c|l|c|c|c|}
\hline \multirow{2}{*}{ parameter meaning } & \multicolumn{3}{|c|}{ units } \\
\cline { 3 - 5 } & & $\boldsymbol{B}_{\boldsymbol{m}}$ & $\boldsymbol{T} \boldsymbol{F}$ & $\boldsymbol{c}_{\boldsymbol{n}}$ \\
\hline $\boldsymbol{\gamma}$ & geometric field error & $(\mathrm{Tm} / \mathrm{kA})$ & $(\mathrm{Tm} / \mathrm{kA})$ & $($ units $)$ \\
\hline $\boldsymbol{\mu}$ & d.c. magnetization strength & $(\mathrm{Tm} / \mathrm{kA})$ & $(\mathrm{Tm} / \mathrm{kA})$ & $($ unit $)$ \\
$\boldsymbol{p}$ & d.c. magnetization pinning exponent & $(-)$ & $(-)$ & $(-)$ \\
$\boldsymbol{q}$ & d.c. magnetization pinning exponent & $(-)$ & $(-)$ & $(-)$ \\
$\boldsymbol{h}$ & d.c. magnetization pinning exponent & $(-)$ & $(-)$ & $(-)$ \\
\hline $\boldsymbol{\sigma}$ & iron saturation strength & $(\mathrm{Tm} / \mathrm{kA})$ & $(\mathrm{Tm} / \mathrm{kA})$ & $($ units $)$ \\
$\boldsymbol{I}_{\boldsymbol{\theta}}$ & iron saturation current & $(\mathrm{A})$ & $(\mathrm{A})$ & $(\mathrm{A})$ \\
$\boldsymbol{S}$ & iron saturation current range & $(-)$ & $(-)$ & $(-)$ \\
\hline $\boldsymbol{\rho}$ & residual magnetization strength & $(\mathrm{Tm} / \mathrm{kA})$ & $(\mathrm{Tm} / \mathrm{kA})$ & $($ units $)$ \\
$\boldsymbol{r}$ & residual magnetization exponent & $(-)$ & $(-)$ & $(-)$ \\
\hline
\end{tabular}

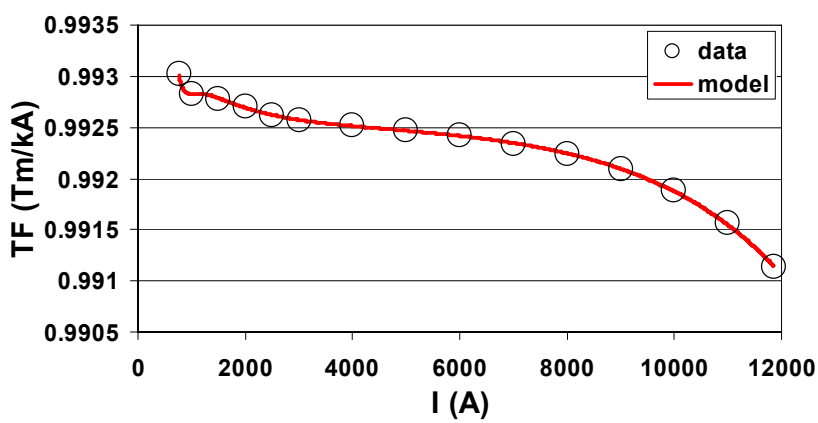

Figure 1: Average of the quadrupoles' main field (ramp up) measured and the static model fit with a max error equal to 0.01 units (measurement's uncertainty $\sim 5$ units for the transfer function).

The following table summarizes the fitting parameters found for the average of the static transfer functions of about 440 main dipole apertures and 80 main quadrupole apertures measured at cold.

Table 2: Summary of the fitting parameters found for the static

\begin{tabular}{|c|c|c|c|c|c|c|c|}
\hline $\begin{array}{c}2- \\
\text { poles }\end{array}$ & TF & $\mathbf{b}_{3}$ & $b_{5}$ & $\begin{array}{c}\text { 4- } \\
\text { poles }\end{array}$ & TF & $b_{6}$ & $b_{10}$ \\
\hline$\gamma$ & 10.1174 & 3.671 & -0.136 & $\gamma$ & 0.9925 & 3.287 & -0.197 \\
\hline$\mu$ & -0.0032 & -7.321 & 0.796 & $\mu$ & -0.1900 & -3.907 & 0.055 \\
\hline$p$ & 0.3820 & 0.414 & 0.114 & $p$ & -1.6317 & 0.408 & -4.042 \\
\hline$q$ & 0.4565 & 0.487 & 0.098 & $q$ & 1.3157 & -0.157 & 0.399 \\
\hline$h$ & 2.000 & 2.000 & 2.000 & $h$ & 2.000 & 2.000 & 2.000 \\
\hline$\sigma^{I}$ & -0.5436 & 0.332 & -0.093 & $\sigma^{I}$ & -0.5604 & 1.484 & -0.071 \\
\hline$I_{0}{ }^{l}$ & 13466 & 2236 & 8578 & $I_{0}{ }^{1}$ & 22463 & 11710 & 5146 \\
\hline$S^{1}$ & 3.2287 & 12.758 & 7.994 & $S^{1}$ & 10.5100 & 23.88 & 4.409 \\
\hline$\sigma^{2}$ & 0.2577 & 0.921 & -0.049 & $\sigma^{2}$ & 0.0510 & -0.921 & 0.211 \\
\hline$I_{0}{ }^{2}$ & 10846 & 11497 & 11499 & $I_{0}{ }^{2}$ & 17555 & 11594 & 12878 \\
\hline$S^{2}$ & 1.7425 & 37.053 & 18.407 & $S^{2}$ & 1.1183 & 2.711 & 0.751 \\
\hline $\bar{\rho}$ & 0.0070 & 1.366 & 0.188 & $\bar{\rho}$ & 0.2086 & 1.276 & -0.068 \\
\hline$r$ & 1.4860 & 4.691 & 1.109 & $r$ & 3.7043 & 4.467 & 0.279 \\
\hline
\end{tabular}
field model for the dipoles and quadrupoles measured at cold.

The maximum error of the fits was below 0.2 units for the transfer function and below 0.03 units for the multipoles; the max errors required for the model are 5 and 0.1 units respectively. The model of the magnetic field therefore provides a powerful tool to predict the reproducible current dependent magnetic state of the LHC during its operation. In addition to the static field model, the dynamic field model is based on the understanding of the magnetic effects which are dependent on both current and time. It is based on dedicated magnetic measurements in cold conditions using the rotating coils measurement system. The main contribution to the dynamic field model is the decay of the magnetization $\left(c_{n}^{\text {decay }}\right)$ that is an effect 
due to current redistributions in the superconducting cables. It manifests itself as a drift in time of the main field and of the multipoles, and is important during beam injection and whenever the current is kept constant at low field. The magnitude of the decay depends on the waveform and waiting time of previous cycles thus making this effect non-reproducible from cycle to cycle. In this paper the decay of the magnetic field is measured for $1000 \mathrm{~s}$ on the injection plateau $(760 \mathrm{~A})$ after a standard pre-cycle [4] with a current ramp rate of $50 \mathrm{~A} / \mathrm{s}$. The normalized decay can be modeled by the following equation:

$\Delta\left(t, t_{i n j}, \tau, d\right)=d\left(1-e^{\frac{t_{i j j}-t}{\tau}}\right)+(1-d)\left(1-e^{\frac{t_{i n j}-t}{9 \tau}}\right)$

which holds for $I=I_{i n j}, t>t_{i n j} . t$ is the instantaneous time, $t_{i n j}$ is the time when injection starts, $I_{i n j}$ is the current at injection, and $\tau$ is the time constant of the decay. The parameter $d$ gives the normalized weight of the fast mode of the decay while its complement to one, $1-d$, gives the normalized weight of the slow mode. Under the hypothesis that the cable current distributes continuously among the strands of a uniform cable, the time evolution of the current is governed by an infinite series of harmonic modes damped by an exponential with a time constant $\tau_{n}=\tau /(2 n-1)^{2}$ [5]. Eq. 1 is limited to the first two modes. The contribution of the decay to the transfer function and to the harmonics is modeled by:

$$
c_{n}^{\text {decay }}=\delta_{n} \frac{\Delta\left(t, t_{i n j}, \tau_{n}, d_{n}\right)}{\Delta\left(t_{i n j}^{s t d}, t_{i n j}, \tau_{n}, d_{n}\right)} \cdot \frac{I_{i n j}}{I},
$$

where the parameter $\delta_{n}$ represents the decay amplitude at a reference time $t^{\text {std }}$. Fig. 2 shows an example of the modeling of the decay of a main quadrupole after $1000 \mathrm{~s}$ at injection current $(760 \mathrm{~A})$. The rms of the fit of this example is 0.01 units, while the maximum error is 0.03 units, comparable with the reproducibility of the rotating coil measurement system $(\sim 0.01$ units for the multipoles).

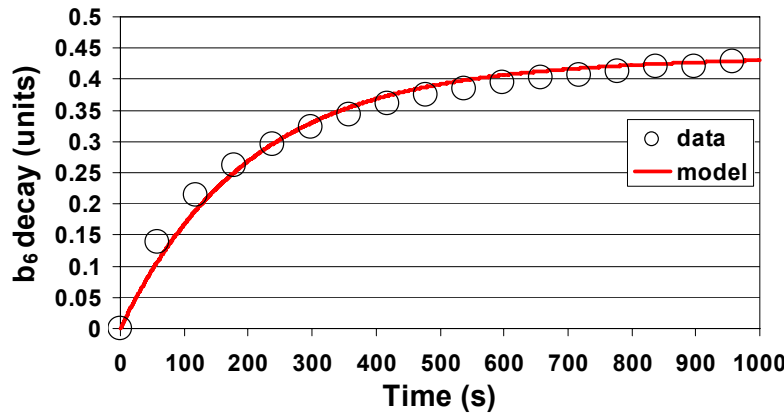

Figure 2: The decay of the $b_{6}$ of a main quadrupole at injection current plateau of $1000 \mathrm{~s}$ calculated with a standard pre-cycle.

Table 3 and 4 show the decay amplitude measured while Table 5 summarizes all the parameters obtained fitting the decay with Eq. 1 for the main dipoles and quadrupoles.
Table 3: The dipoles' average decay amplitude measured for the $b_{1}, b_{3}$ and $b_{5}$ after $1000 \mathrm{~s}$ at injection current (760 A).

\begin{tabular}{|c|c|c|c|}
\hline & $\begin{array}{c}\mathbf{b}_{1} \\
\text { [units] }\end{array}$ & $\begin{array}{c}\mathbf{b}_{\mathbf{3}} \\
\text { [units] }\end{array}$ & $\begin{array}{c}\mathbf{b}_{\mathbf{5}} \\
\text { [units] }\end{array}$ \\
\hline $\begin{array}{c}\text { Dipole average decay amplitude } \\
\text { after 1000 s }(\boldsymbol{\delta})\end{array}$ & 1.54 & 1.99 & -0.34 \\
\hline $\begin{array}{c}\text { Dipole standard deviation of the } \\
\text { decay amplitude after 1000 s }\end{array}$ & 0.74 & 0.45 & 0.11 \\
\hline
\end{tabular}

Table 4: The quadrupoles' average decay amplitude measured for the $b_{2}$ and the $b_{6}$ after $1000 \mathrm{~s}$ at injection current $(760 \mathrm{~A})$.

\begin{tabular}{|c|c|c|}
\hline & $\begin{array}{c}\mathbf{b}_{\mathbf{2}} \\
\text { [units] }\end{array}$ & $\begin{array}{c}\mathbf{b}_{\mathbf{6}} \\
\text { [units] }\end{array}$ \\
\hline $\begin{array}{c}\text { Quadrupole average decay amplitude } \\
\text { after 1000 s }(\boldsymbol{\delta})\end{array}$ & -3.09 & 0.59 \\
\hline $\begin{array}{c}\text { Quadrupole standard deviation of the } \\
\text { decay amplitude after 1000 s }\end{array}$ & 1.19 & 0.39 \\
\hline
\end{tabular}

Table 5: The averages of the dipoles and quadrupoles fitting parameters found for the decay amplitude after $1000 \mathrm{~s}$ at injection current $(760 \mathrm{~A})$. The standard deviation (Std) is also given.

\begin{tabular}{|c|c|c|c|c|c|c|}
\hline & $\begin{array}{c}\boldsymbol{\tau} \\
{[\mathrm{s}]}\end{array}$ & $\begin{array}{c}\text { Std } \boldsymbol{\tau} \\
{[\mathrm{s}]}\end{array}$ & $\begin{array}{c}\boldsymbol{d} \\
{[-]}\end{array}$ & $\begin{array}{c}\text { Std } \boldsymbol{d} \\
{[-]}\end{array}$ & $\begin{array}{c}\text { rms } \\
{[\text { units] }}\end{array}$ & $\begin{array}{c}\text { Std rms } \\
{[\text { units] }}\end{array}$ \\
\hline 2-pole $\mathbf{b}_{\mathbf{1}}$ & 54.1 & 40.1 & 0.27 & 0.13 & 0.17 & 0.05 \\
\hline 2-pole $\mathbf{b}_{\mathbf{3}}$ & 40.2 & 7.2 & 0.23 & 0.07 & 0.01 & $5.3 \mathrm{E}-03$ \\
\hline 2-pole $\mathbf{b}_{\mathbf{5}}$ & 46.9 & 15.7 & 0.23 & 0.08 & $2.2 \mathrm{E}-03$ & $1.3 \mathrm{E}-03$ \\
\hline 4-pole $\mathbf{b}_{\mathbf{2}}$ & 42.2 & 26.4 & 0.29 & 0.13 & 0.27 & 0.05 \\
\hline 4-pole $\mathbf{b}_{\mathbf{6}}$ & 44.3 & 8.8 & 0.19 & 0.07 & $3.8 \mathrm{E}-03$ & $1.5 \mathrm{E}-03$ \\
\hline
\end{tabular}

The RMS values of the fits applied on the decay of the magnetic field measured after a standard pre-cycle are below 0.15 units. This makes the model suitable for the prediction of the field errors.

\section{CONCLUSIONS}

We have developed a parametric field model to forecast the magnets' field errors in the LHC machine. The parameters of the models were extracted from cold magnetic measurements and were divided in static and dynamic field components. The model was applied to the behavior of about 440 main dipole apertures and 80 main quadrupole apertures and the maximum error was shown to be well within the targeted accuracy. In the next months the model will be qualified using dipoles and quadrupoles and consequently fine-tuned to take into account the powering history dependence of the magnetic field of all types of LHC magnets.

\section{REFERENCES}

[1] E. Todesco, "Estimates of the LHC Magnetic Optics Versus Requirements", LHC project workshop, 'Chamonix XIV'.

[2] L. Bottura et al., "A Strategy for Sampling the Field Quality of the LHC Dipoles, “EPAC’04, Lucerne, June 2004.

[3] J. Billan, L. Bottura et al., "Twin rotating coils for cold magnetic measurements of $15 \mathrm{~m}$ long LHC dipoles", IEEE Transactions on Applied Superconductivity, March 2000.

[4] N. Sammut, "The Field Description for The Large Hadron Collider", Ph.D. Thesis, Malta 2006.

[5] L. Bottura, M. Breschi, M. G. Fabbri, "Analytical Solution for the Current Distribution in Multistrand Superconducting cables", Journal of Applied Physics, vol. 92, December 2002. 